\title{
ESTUDIO DE DIVERSAS ESPECIES DE LOS GÉNEROS TRACHELOMONAS Y STROMBOMONAS (EUGLENOPHYTA)
}

\author{
Julio Carlos DE LA ROSA \& Pedro M. SÁNCHEZ CASTILLO
}

\begin{abstract}
RESUMEN: Se estudian siete especies de Euglenofíceas loricadas, 2 pertenecientes al género Strombomonas y 5 de Trachelomonas. Se analiza la microarquitectura de la lórica a microscopio óptico y electrónico de barrido, describiendo los elementos ornamentales de cada especie. Destacamos el estudio de tres poblaciones de Trachelomonas, dos de ellas incluídas provisionalmente en $T$. nigra y $T$. similis respectivamente, mientras que en la tercera: Trachelomonas sp., con una gran variabilidad morfológica de la lórica, destaca la existencia de un anillo de perforaciones que se disponen alrededor del poro flagelar.
\end{abstract}

Palabras clave: Algas, Euglenofíceas, Trachelomonas, Strombomonas.

SUMMARY: Seven species of Euglenophyceae with lorica (2 Strombomonas and 5 Trachelomonas) haven been studied. We analyzed their lorica microarchitecture by means of ligh microscopy and scanning electron microscopy, enumerating their different ornamental elements. We stand out the study of three population of Trachelomonas, two of them are provisionally included in T. nigra and T. similis respectively. The third one: Trachelomonas $s p$. show a large morphological variability with only one common ornamental character within of the population: a ring of perforations around the flagellum pore.

Key words: Algae, Euglenoids, Trachelomonas, Strombomonas.

\section{INTRODUCCIÓN}

La descripción de los táxones incluidos en los géneros Trachelomonas Ehrenberg y Strombomonas Deflandre se ha basado tradicionalmente en la morfología, tamaño y ornamentación de la lórica. La especial importancia que juega la ornamentación en el concepto de especie en estas euglenofíceas loricadas hace muy aconsejable la utilización de la microscopía electrónica de barrico (MEB) para su exacta descripción. 
Los estudios realizados a MEB han dado una base ultraestructural más sólida a las clásicas observaciones de microscopía óptica y han aportado una mayor información, por ello, y a la luz de los trabajos llevados a cabo por diferentes autores (Rosowski et al., 1975; Tell \& Conforti, 1984; Conforti \& Tell, 1986, 1989) consideramos necesario el estudio del mayor número posible de especies con esta técnica, para poder aportar más datos que ayuden a clarificar el valor sistemático de la lórica y de su ornamentación. Posiblemente, la utilización generalizada de la microscopía electrónica, permita abordar el estudio definitivo de Trachelomonas y Strombomonas, ya que como indican Tell \& Conforti (1984) «si bien hay caracteres que nos inducen a pensar en una separación entre ambos géneros, la frontera entre ellos es difícil de definir».

\section{MATERIAL Y MÉTODOS}

El material estudiado procede de poblaciones naturales, desarrolladas tanto en el plancton como en el herpon, de lagunas someras de reducido volumen. Un grupo de lagunas (las de los Prados de Otero, Sierra Nevada, Granada) se sitúan sobre los $2500 \mathrm{~m}$ de altitud y sus cuencas son silíceas. Las otras lagunas (Charco Negro y Alcantar, Sierra de Loja, Granada) se sitúan sobre los 1500 m y su cuenca es caliza. Las muestras fueron recogidas y fijadas in situ con formaldehído al 3-4\%. Para su observación al microscopio electrónico de barrido las células fueron aisladas por sedimentación y deshidratadas. Tras la deshidratación se cubrieron con una película de oro-paladio en alto vacío. La observación se llevó a cabo en un microscopio Zeiss DSM 950 de los Servicios Técnicos de la Universidad de Granada.

Para la descripción de la ornamentación de la lórica hemos seguido la propuesta por Conforti \& Tell (1986).

\section{RESULTADOS Y DISCUSIÓN}

Strombomonas scabrae (Playf.) Tell \& Conforti (Lám. 1, Fig. 1; Lám. 2, Fig. 1).

Lórida oboidal, adelgazada en la parte final y truncada en el ápice. Cuello no muy diferenciado, de 6 a $7 \mu \mathrm{m}$ de ancho y de borde irregular. Lórica marrón amarillenta, raras veces aparece hialina.

A MEB la superficie de la lórica está constituida por numeosas concreciones de partículas que recubren toda su superficie y le dan un aspecto irregular y escábrido. El tamaño de las concreciones es muy variable. Dim: 17,2-18,2 ( $x=18)$ x 24-25 ( $x=24,7) \mu \mathrm{m}$.

Loc.: Charco Negro, Sierra de Loja, Granada.

Strombomonas verrucosa (Daday) Defl. (Lám. 2, Fig. 2)

Lórica caracterizada por estrecharse hacia la zona media, formando una cintura marcada de anchura variable, entre 15 y $23 \mu \mathrm{m}(\mathrm{x}=17,3 \mu \mathrm{m})$. Hacia la parte anterior se estrecha progresivamente en un cuello cilíndrico recto u oblícuo ( $\mathrm{x}=5,6 \mu \mathrm{m}$ de diámetro). La mitad posterior de la lórica, a partir de la cintura, se ensancha alcanzando el máximo grosor ( $x=22,1$ 


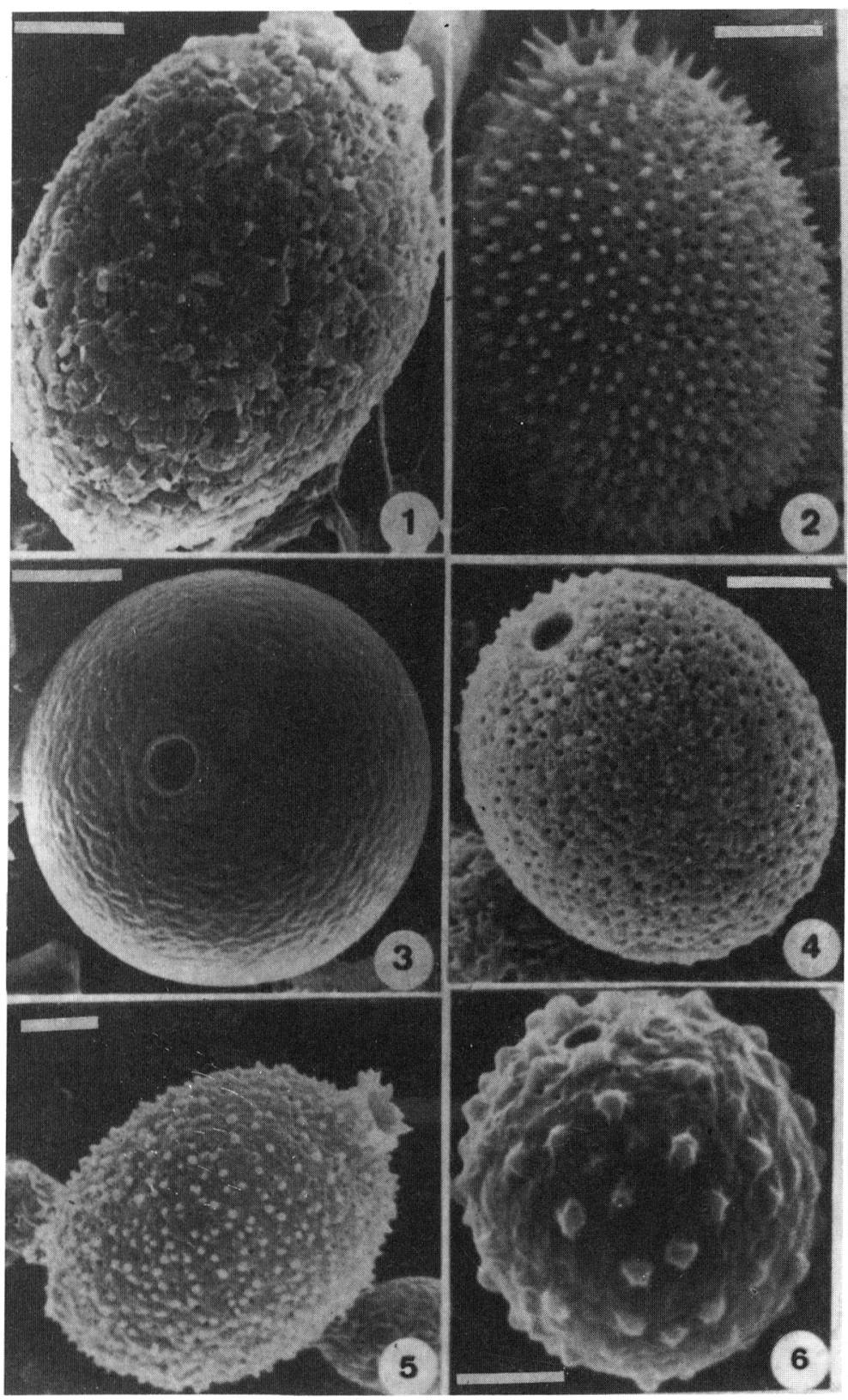

Lámina 1. Fig. 1. Strombomonas scabrae. Fig. 2. Trachelomonas hipida. Fig. 3. Trachelomonas nexilis. Fig. 4.Trachelomonas cf. nigra. Fig. 5. Trachelomonas cf. similis. Fig. 6. Trachelomonas sp. 
$\mu \mathrm{m})$ y estrechándose bruscamente en una pequeña prolongación que puede llegar a medir hasta $6 \mu \mathrm{m}$ de longitud. Lórica siempre hialina. Dim.: 26-32 (x=27,7) x 22-22,8 (x=22,1) $\mu \mathrm{m}$. Este taxon se asemeja por su morfología a S. acuminata (Schmarda) Defl., de la que nosotros la hemos diferenciado debido a su menor tamaño y distinta ornamentación.

De acuerdo con Tell \& Conforti (1984), observamos que la superficie externa de la lórica tiene la capacidad de aglutinar partículas. Esta propiedad puede ser la responsable del monótono tipo de ornamentación que muestran las especies de este género a M.O.

Loc.: Charco Negro, Sierra de Loja, Granada.

Trachelomonas hispida (Pertg) Stein (Lám. 1, Fig. 2; Lám. 2, Fig. 3).

Lórica elipsoidal, espinosa y con un cuello formado por espinas dispuestas de forma concéntrica claramente diferenciadas.

A MEB se observan distintos ornamentos. El más aparente es espinoso, constituido por espinas cortas y cónicas, uniformemente repartidas por toda la superficie y de hasta $1.9 \mu \mathrm{m}$ de altura. El segundo tipo está constituído por perforaciones también repartidas uniformemente y de diámetro inferior a $0,3 \mu \mathrm{m}$. El cuello está poco desarrollado y formado por espinas típicas que se sueldan en su tercio basal. Dim.: 25,9-27,8 ( $x=27,3)$ x 17,2-19,2 ( $x=17,9) \mu \mathrm{m}$.

Loc.: Laguna de Otero, Sierra Nevada, Granada.

Trachelomonas nexilis Palmer (Lám. 1, Fig. 3; Lám 2, Fig. 4).

Lórica esférica. Espesamiento anular variable formado desde por un simple reborde, hasta por un corto tubo. Diámetro del poro de hasta $1,9 \mu \mathrm{m}$ de diámetro, en todos los casos incluido en una leve depresión de la lórica. Flagelo de 2 a 3 veces más largo que la célula. Pared rugosa. Lórica coloreada marrón-amarillenta.

A MEB se observa que la ornamentación de la lórica está constituida por depresiones vermiculosas, de escasa profundidad y anastomosadas unas con otras. Dim: 14,1-19,2 $(x=17,6)$.

Conforti \& Tell (1986) dan unas dimensiones de $19 \times 17 \mu \mathrm{m}$ sobre el material recolectado en la república de Argentina. El estudio ultraestructural de Tr. nexilis llevado a cabo por estos autores, coincide en líneas generales con nuestras observaciones, si bien la ornamentación es más patente que en nuestros ejemplares.

Loc.: Laguna de Alcantar, Sierra de Loja, Granada.

Trachelomonas ef. nigra Swir (Lám. 1, Fig. 4; Lám. 2, Fig. 5).

Lórica elipsoidal, con poro flagelar sencillo, sin cuello. Flagelo de dos a tres veces más largo que la célula. Lórica finamente ornamentada marrón-amarillenta.

A MEB la ornamentación es heterogénea. Un tipo ornamental es punteado, con perforaciones repartidas por toda la superficie de la célula $\left(4,8 \mu \mathrm{m}^{2}\right)$ de diámetro inferior a 0,3 $\mu \mathrm{m}$. El segundo es papiloso constituido por dos tipos de papilas, unas muy pequeñas y de distribución muy irregular y otras mayores restringidas a la zona apical de la célula $(5,8 / 5$ $\mu \mathrm{m}^{2}$ ) de hasta $1,4 \mu \mathrm{m}$ de altura y que constituyen un anillo concéntrico alrededor del poro flagelar. Dim.: 16,3-18,2 (x=18,1) x 19,6-29,5 (x=19,8) $\mu \mathrm{m}$.

La descripción morfológica a microscopía óptica de nuestros ejemplares coincide en líneas generales con Trachelomonas nigra, sin embargo no disponemos de datos sobre la ultraestructura de este taxon. Conforti \& Tell (1986) describen una Trachelomonas sp. (Págs. 60-61) muy semejante al material estudiado, pero con un cuello más desarrollado.

Loc.: Charco Negro, Sierra de Loja, Granada. 


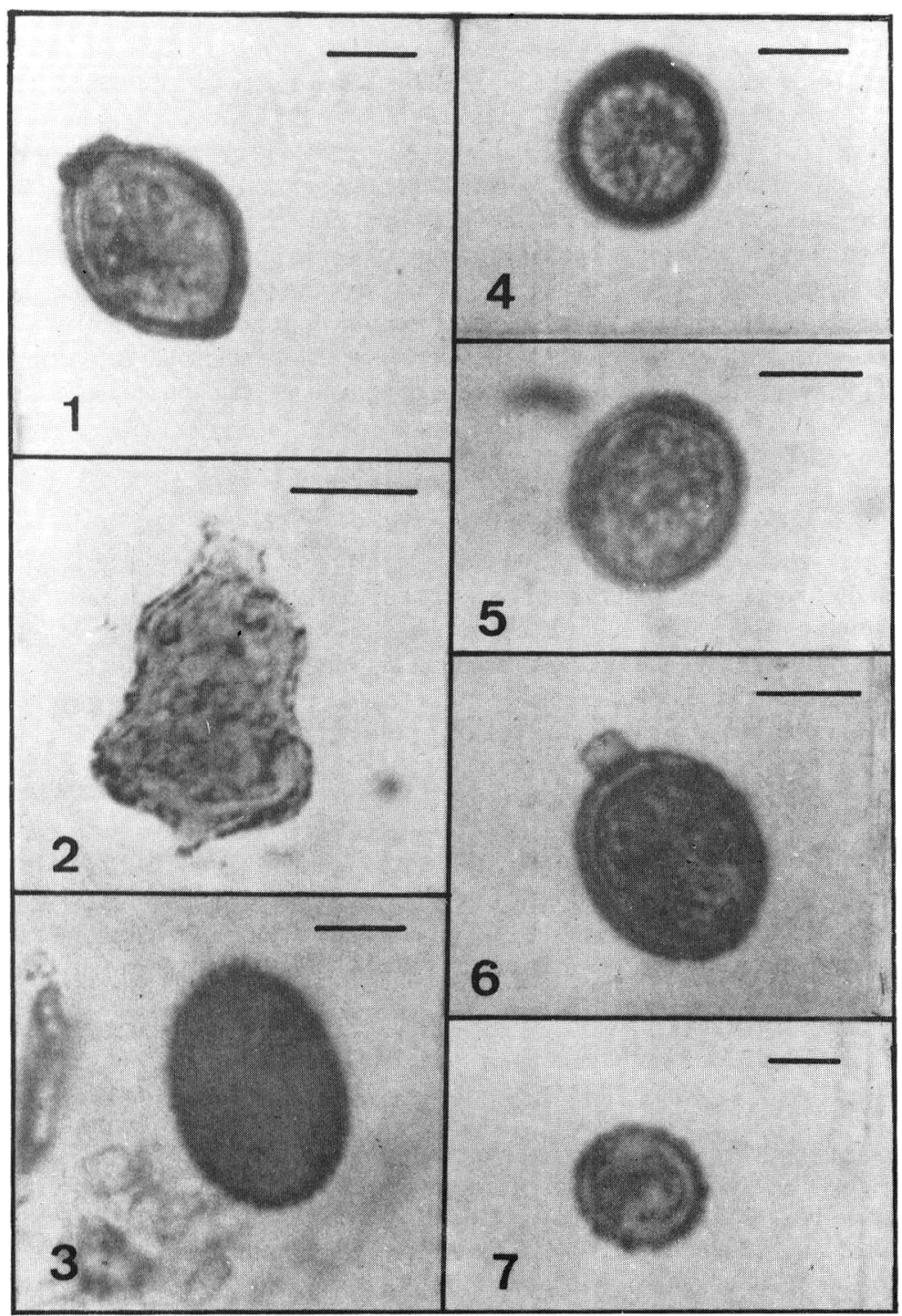

Lámina 2. Fig. 1 Strombomonas scabrae. Fig. 2. Strombomonas verrucosa. Fig. 3. Trachelomonas hispida. Fig. 4. Trachelomonas nexilis. Fig. 5. Trachelomonas cf. nigra. Fig. 6. Trachelomonas cf. similis. Fig. 7. Trachelomonas sp. (Todas las escalas representan $10 \mu \mathrm{m}$ ) 
Trachelomonas cf. similis Swir (Lám. 1, Fig. 5; Lám. 2, Fig. 6).

Lórica elipsoidal, formando en la parte apical un cuello corto y ligeramente curvado, cilíndrico, bien diferenciado del resto del cuerpo. Flagelo de 2 a 3 veces más largo que la longitud de la célula. Lórica papilosa y coloreada marrón-amarillenta.

A MEB la lórica aparece con una ornamentación heterogénea. Presenta papilas y espinas bacilares, unas pequeñas y muy numerosas y las otras de mayor tamaño, también abundantes $\left(7,5 / 5 \mu \mathrm{m}^{2}\right)$ pudiendo llegar a medir hasta $1 \mu \mathrm{m}$ de altura. Un tercer tipo ornamental lo forman puntuaciones de diámetro inferior a $0,5 \mu \mathrm{m}$ y repartidas por toda la superficie de la lórica $\left(5,4 / 5 \mu \mathrm{m}^{2}\right)$. El cuello se abre en un reborde engrosado donde se localizan espinas cónicas de mayor tamaño (hasta $2 \mu \mathrm{m}$ de altura) por lo que adquiere aspecto denticulado. Dim.: 17-20 (x=18,6) x 21,1-23 (x=22,3) $\mu \mathrm{m}$.

El tamaño de las espinas bacilares es muy reducido por lo que sólo son claramente observables a MEB, ya que a microscopio óptico se pueden confundir con otros elementos (perforaciones o papilas). Los caracteres de forma de la lórica (elipsoidal) y del cuello (ligeramente curvado), nos hacen pensar en la inclusión de esta población en Trachelomonas similis, si bien el grado de curvatura del cuello, su tamaño y el tamaño celular no coinciden plenamente.

Loc.: Charco Negro, Sierra de Loja, Granada.

Trachelomonas sp. (Lám. 1, Fig. 6; Lám. 2, Fig. 7).

Lórica subcircular o semielíptica. Flagelo hasta dos veces la longiud de la célula. Poro flagelar rodeado por una estructura que puede estar desde poco diferenciada hasta constituida por gruesas verrugas. Ornamentación de la lórica muy variable, observándose desde ligeramente rugosa hasta groseramente verrucosa. Coloración marrón-amarillenta a rojiza.

En la descripción a MEB destaca la existencia de una serie de poros que forman una estructura circular concéntrica al poro flagelar y que a M.O. aparecen como puntos mas refringentes. Dim: 18,1-19,1 x 16,3-17,2 $\mu \mathrm{m}$.

Loc.: Laguna de Otero, Sierra Nevada, Granada.

\section{BIBLIOGRAFÍA}

CONFORTI, V. \& G. TELL -1986- Ultraestructura de la lórica de Trachelomonas Def. (Euglenophyta) en microscopio electrónico de barrido (MEB). Nova Hedwigia, 43(1-2):4579.

CONFORTI, V. \& G. TELL -1989- Ultraestructure of the pellicle and the envelope of some euglenoid flagellates from Argentina by means of S.E.M. Nova Hedwigia, 48(1-2):187206.

ROSOWSKI, J.R., R.L. VADAS \& P. KRUGENS -1975- Surface configuraions as realted with Scanning Electron Mycroscopy. Ann. J. Bot., 62(1):48-57.

TELL, G. \& V. CONFORTI -1984- Ultraestructura de la lórica de cuatro especies de Strombomonas Def. (Euglenophyta) en MEB. Nova Hedwigia, 40:123-132.

TELL, G. \& V. CONFORTI -1988- Quelques Strombomonas Def.(Euglonophyta) de l'Argentine au microscope pnotonique et élèctronique à balayage. Nova Hedwigia, 46(34):541-556

(Aceptado para su publicación en Junio de 1.990)

Dirección de los autores: Departamento de Biología Vegetal. Facultad de Ciencias. Universidad de Granada. 